\title{
A study of blow-ups in the Keller-Segel model of chemotaxis
}

\author{
Ibrahim Fatkullin \\ Department of Mathematics, University of Arizona, USA
}

Received 3 May 2012, in final form 1 October 2012

Published 16 November 2012

Online at stacks.iop.org/Non/26/81

Recommended by A L Bertozzi

\begin{abstract}
We study the Keller-Segel model of chemotaxis and develop a composite particle-grid numerical method with adaptive time stepping which allows us to resolve and propagate singular solutions. We compare the numerical findings (in two dimensions) with analytical predictions regarding formation and interaction of singularities obtained through analysis of the stochastic differential equations associated with the model.

PACS numbers: $02.60 . \mathrm{Nm}, 02.70 . \mathrm{Ns}, 05.10 . \mathrm{Gg}, 87.10 . \mathrm{Ed}, 87.10 . \mathrm{Mn}$, 87.17.Jj

Mathematics Subject Classification: 35K58, 45G05, 65C35, 82C22, 82C31, $82 \mathrm{C} 80,92 \mathrm{C} 17$
\end{abstract}

\section{Introduction}

In this work we discuss a numerical method for studying singularities (blow-ups) in the KellerSegel (K-S) model of chemotaxis [31] and in the systems with similar mathematical structure, such as the McKean-Vlasov (McK-V) model of charged interacting particles [46]. (Patlack derived similar equations nearly two decades prior to Keller and Segel [34].) We present illustrative numerical examples and provide analytical insights into formation and interaction of singularities from the point of view of the associated stochastic processes. We concentrate on the details of numerical modelling and several specific aspects of mathematical analysis. An interested reader should consult the exhaustive reviews for discussions of physical and biochemical phenomena involved in chemotaxis [27, 28, 41,42].

Numerical treatment of equations with singular solutions is challenging because it requires a reliable and efficient approximation of singular functions or even distributions. For example, the conventional finite-difference or spectral methods are not fit for dealing with $\delta$-functions arising in the K-S model. While most finite-element and discontinuous Galerkin methods are efficient and accurate as long as the solutions remain sufficiently smooth, in the vicinity of singularities they struggle with positivity issues, unphysical oscillations, and related 
problems [20-23, 38, 39]. (A conservative upwind finite-element method introduced by Saito for the elliptic K-S model overcomes some of these issues [35].) Generally, unless some regularizations are introduced, these methods cannot propagate solutions past the moment of blow-up altogether. The method presented in this work avoids such difficulties because the singular field is modelled by an ensemble of interacting particles. The singularities manifest themselves as particle aggregates, harmless from the numerical standpoint. Essentially, this approach to chemotaxis reverses the continuous PDE description of ensembles of interacting particles. Such methods for sufficiently well-behaved nonlinear integro-differential equations have been studied by Bossy and Talay [9,10]. Haškovec and Schmeiser have recently introduced a method utilizing similar ideas [24,25]. Their method, however, is exclusively particle-based and is limited to one specific variety of the elliptic $\mathrm{K}-\mathrm{S}$ model in the entire plane. Our method employs the grid and particle representations of the fields simultaneously and is suitable for both elliptic and parabolic $\mathrm{K}-\mathrm{S}$ equations; it does not require an explicit knowledge of Green's function in a specific domain. It is also applicable to a wider range of kinetic problems. (Other technical differences are discussed where appropriate.) Conceptually, our method is more in the spirit of the particle-in-cell (PIC) methods widely used, e.g., in plasma physics $[1,5,16,26,29,45,47]$. Another interesting numerical method has been recently implemented for the one-dimensional $\mathrm{McK}-\mathrm{V}$ system [6]. It uses that the $\mathrm{McK}-$ $\mathrm{V}$ equation describes steepest descent dynamics in the Wasserstein-2 space of probability measures and is suitable for studying singular (measure-valued) solutions.

The usefulness of the particle approach extends beyond numerical methods. Analysis of the stochastic processes underlying the K-S PDEs yields insights into formation and interaction of singularities. In particular, we derive an expression for the critical mass required to create or sustain a singularity and relate the non-uniqueness of solutions of the $\mathrm{K}-\mathrm{S}$ equations to the underlying diffusion process. Even though our treatment is rather informal, it sheds new light on the mechanism of blow-ups. Some of the results presented in this paper have been proven using different methods and under various assumptions. Much effort has been directed towards obtaining the exact value of the critical mass $[4,8,11,13,17,18,32]$. Velázquez introduced measure-valued solutions and extended the $\mathrm{K}-\mathrm{S}$ equations beyond the point of blow-up [43,44], see also [19]. These works together with the study of the K-S equations as a hydrodynamic limit of interacting particle systems by Stevens [37] link the particle and PDE descriptions of chemotaxis. We also mention a rigorous analysis of phase transitions in the $\mathrm{McK}-\mathrm{V}$ system [15] (it only covers sufficiently regular interaction kernels not relevant to the $\mathrm{K}-\mathrm{S}$ model) and an investigation of singularity formation in several related aggregation models $[2,3,12]$ (these models do not have diffusive terms and correspond to one special case of the $\mathrm{K}-\mathrm{S}$ model).

The $K-S$ model is prescribed by the following system of PDEs:

$$
\begin{aligned}
& \partial_{t} \rho(\boldsymbol{x}, t)=\nabla \cdot(\mu \nabla \rho-\chi \rho \nabla c), \\
& \alpha \partial_{t} c(\boldsymbol{x}, t)=\Delta c-k^{2} c+\rho .
\end{aligned}
$$

The function $\rho(\boldsymbol{x})$ is the density of active particles (bacteria), $c(\boldsymbol{x})$ is the concentration of chemoattractant. For numerical simulations we use Neumann (no flux) boundary conditions,

$$
\partial_{n} c(\boldsymbol{x})=\partial_{n} \rho(\boldsymbol{x})=0 \quad \text { for } \quad \boldsymbol{x} \in \partial \Omega
$$

in a two-dimensional square domain $\Omega=(0, L)^{2}$. We assume that $\rho(\boldsymbol{x})$ integrates to $M$ over the entire domain; $M$ is the total mass of the bacteria. By rescaling the equations, we can always achieve that $\alpha=0$ or $\alpha=1$. In the former case the model is called elliptic, in the latter-parabolic. The parameters $\mu, \chi$, and $k$ are constants. (The numerical method, however, may be readily extended to a more general class of equations, see section 2 for details.) 


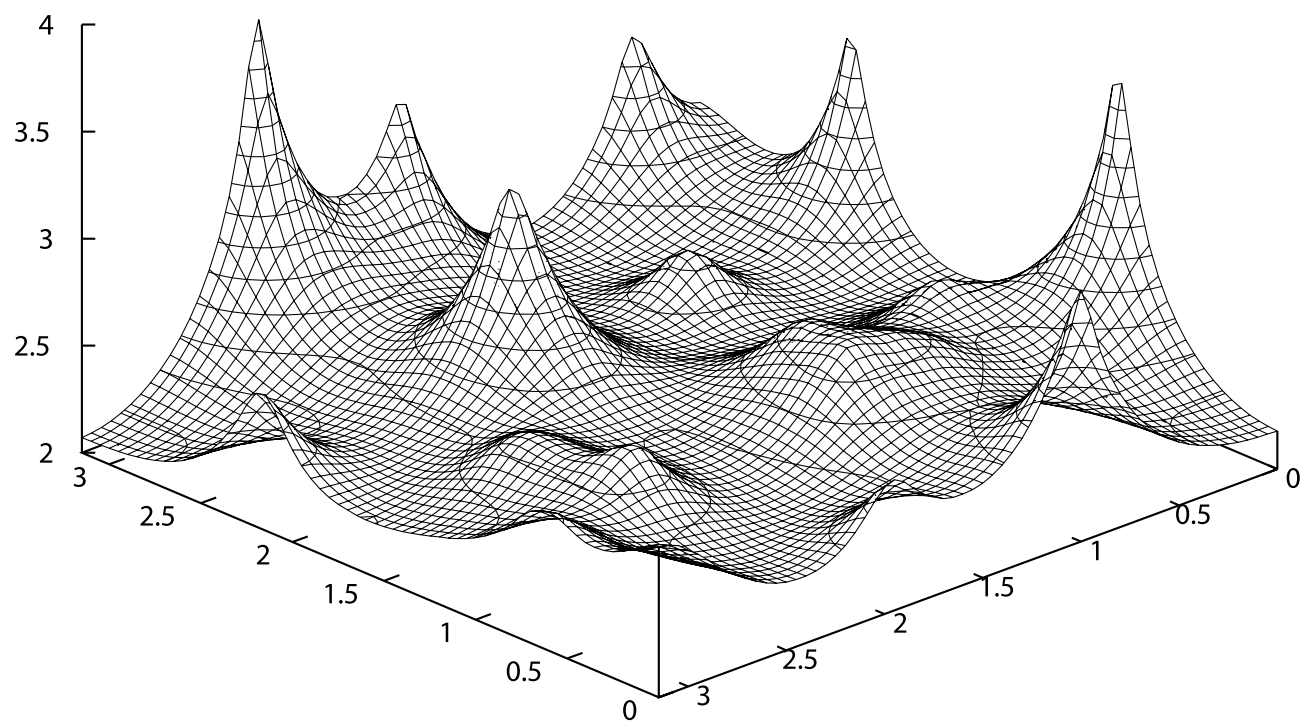

Figure 1. A snapshot $(t=10)$ of the concentration field $c(x)$ illustrating multiple blow-ups in the parabolic model. The parameters are as follows: $\alpha=k=1, \chi=0.1, \mu=0.005, M=25$. The spatial domain, $3.2 \times 3.2$, is discretized using the mesh size $\Delta x=0.05$; the time step for propagation of the concentration field is $\Delta t=0.1 ; 4096$ particles are used to approximate $\rho(\boldsymbol{x})$ (see section 2 for details of the numerical scheme). Some random initial data is used at $t=0$.

Physically, these equations describe the following phenomenon. The chemoattractant spreads diffusively and decays with rate $k^{2}$; it is also produced by the bacteria with rate 1 . (In the elliptic case these rates are infinite, i.e., the chemoattractant 'thermalizes' infinitely fast.) The bacteria diffuse with mobility $\mu$ and also drift in the direction of the gradient of concentration of the chemoattractant with velocity $\chi|\nabla c| ; \chi$ is called chemosensitivity.

In this work we are interested in singular solutions to the $\mathrm{K}-\mathrm{S}$ equations. A typical situation is illustrated in figure 1 where a snapshot of the concentration field $c(x)$ is displayed. The peaks correspond to $\delta$-function-type singularities of the particle density $\rho(\boldsymbol{x})$. (Displaying bacteria density itself is not very illustrative due to its highly singular nature.) As mentioned above, the principal challenge for numerical simulations is in approximating the fields $\rho(\boldsymbol{x})$ and $c(\boldsymbol{x})$ as they become unbounded. Our idea is to use a particle method for the evolution of $\rho(\boldsymbol{x})$, so that its singularities manifest themselves as harmless particle aggregates. For the propagation of $c(x)$, we use a second order implicit finite-difference scheme because of its simplicity and excellent stability properties. Even though $c(x)$ also becomes unbounded analytically (it remains large but bounded for a given discretization), its weak logarithmic singularities do not cause any problems for sufficiently stable schemes. In general, one may use a different solver for propagation of the concentration field (including a particle-based solver as well).

The particle-based description utilizes that the PDE (1a) is a Fokker-Planck equation for the evolution of the probability density of the bacteria. Suppose first, that we are given some time-dependent concentration field $c(\boldsymbol{x}, t)$ and want to solve equation $(1 a)$. Let $\boldsymbol{X}_{t}^{(n)}$ be i. i. d. random variables distributed according to the initial condition $\rho(x, 0)$ at time $t=0$ and satisfying the following stochastic differential equations (with reflecting boundary at $\partial \Omega$ ) for $t>0$ :

$$
\mathrm{d} \boldsymbol{X}_{t}^{(n)}=\chi \nabla c\left(\boldsymbol{X}_{t}^{(n)}, t\right) \mathrm{d} t+\sqrt{2 \mu} \mathrm{d} \boldsymbol{W}_{t}^{(n)}
$$


Then, using the strong law of large numbers and that $(1 a)$ is a Fokker-Planck equation for the SDEs (3), we can see that as $N \rightarrow \infty$, the empirical probability densities,

$$
P_{N}(\boldsymbol{x}, t)=\frac{M}{N} \sum_{n=1}^{N} \delta\left(\boldsymbol{x}-\boldsymbol{X}_{t}^{(n)}\right),
$$

converge almost surely to $\rho(\boldsymbol{x}, t)$. (This convergence is in the sense of measures, point-wise in $t$; a stronger convergence can be established with more effort.) Thus we can simulate equations (3) for the particles, and approximate the density $\rho(x, t)$ using $P_{N}(x, t)$ whenever required.

Rigorously justifying this approach to the full $\mathrm{K}-\mathrm{S}$ system $(1 a)$ and $(1 b)$ is more challenging because in the full system the concentration field $c(\boldsymbol{x}, t)$ is a functional of $\rho(\boldsymbol{x}, s)$, $s \leqslant t$, rather than some a priori prescribed field. This implies that equations (3) become coupled and we end up with a system of stochastic interacting particles (with memory in the parabolic case). Some progress in this direction, nevertheless, has been made, starting with the work of McKean on propagation of chaos [33], see also Sznitman [40]. Stevens derived the $\mathrm{K}-\mathrm{S}$ equations as limit dynamics for interacting stochastic particle systems via smoothing and rescaling the interaction potentials in a particular fashion [37]. Haškovec and Schmeiser proved convergence of their particle method under reasonable assumptions via analysis of the associated BBGKY hierarchy [25].

\section{Numerical method}

As mentioned in the introduction, the principal idea behind our numerical scheme is to employ a particle method for the evolution of the particle density field $\rho(\boldsymbol{x}, t)$. The current implementation utilizes an implicit second order finite-difference scheme for simulating equation $(1 b)$ and an explicit Euler-Maruyama scheme for the stochastic particle dynamics (3). We discretize the computational (rectangular) domain with grid size $\Delta x$ and propagate the concentration field $C_{i j}$ using the time step $\Delta t$. The particle density field $P_{i j}$ is reconstructed from the particle locations. We evolve the particles using adaptive time steps which may be smaller than $\Delta t$; this is needed for stability reasons (see below).

The particle dynamics is simulated using the forward Euler-Maruyama scheme,

$$
\boldsymbol{X}^{(n)}(t+\Delta \tau)=\boldsymbol{X}^{(n)}(t)+\chi \nabla c\left(\boldsymbol{X}^{(n)}(t)\right) \Delta \tau+\sqrt{2 \mu \Delta \tau} \boldsymbol{N}(0,1),
$$

where $N(0,1)$ is a standard Gaussian random variable with mean 0 and variance 1 . We enforce the no-flux Neumann boundary conditions for $\rho(\boldsymbol{x})$ by reflecting the particles escaping from the spatial domain back into it. The gradient field $\nabla c(\boldsymbol{x})$ is approximated in two steps. First, we construct the gradient fields $C X$ and $C Y$ using the second order approximation,

$C X_{i j}=\frac{1}{2 \Delta x}\left[C_{i+1, j}(t)-C_{i-1, j}(t)\right], \quad C Y_{i j}=\frac{1}{2 \Delta x}\left[C_{i, j+1}(t)-C_{i, j-1}(t)\right]$.

Then we approximate $\nabla c(\boldsymbol{x})$ via bilinear interpolation using the values of $C X$ and $C Y$ at the four nearest grid points. Because $|\nabla c(\boldsymbol{x})|$ becomes unbounded (very large numerically) in the vicinity of blow-ups, it is essential to choose the time step $\Delta \tau$ adaptively, see figure 2 and its description for details. Consequently, if needed, we subdivide the timestep $\Delta t$ into smaller intervals $\Delta \tau^{(n)}$ so that $\left|\nabla c\left(\boldsymbol{X}^{(n)}\right)\right| \Delta \tau^{(n)}<\Delta x$. (Each particle is simulated independently of others, i.e. $\Delta \tau^{(n)}$ are chosen for each particle individually.) A failure to satisfy this condition leads to overshooting in the vicinity of blow-ups, which in turn causes artificial oscillations and damping. Knowing that in the direct vicinity of singular points, the concentration field acquires logarithmic singularities, we can deduce that its numerical gradients may get as large 


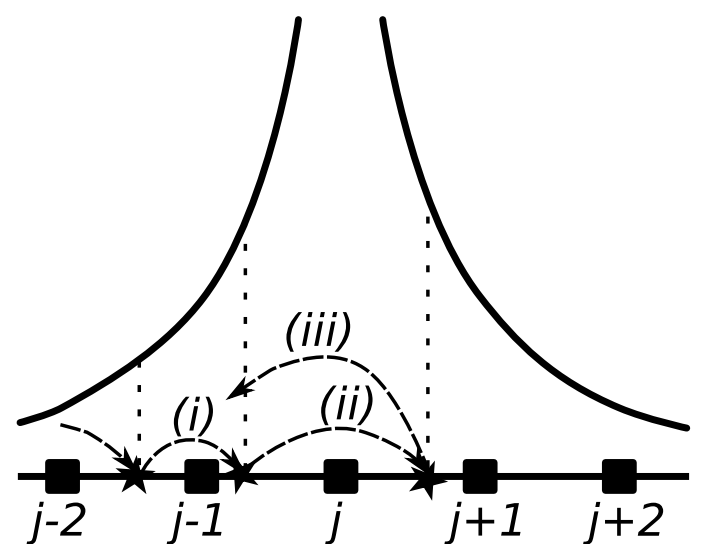

Figure 2. Adaptive time stepping for particle dynamics. In this example a blow-up of the concentration field occurs near a grid point with index $j$. While some particular time step is appropriate for propagating the particles away from the blow-up (i), it may lead to overshooting in its vicinity (ii, iii). Thus the particles incorrectly accumulate near the grid points $j \pm 1$ rather than $j$. One can avoid this phenomenon reducing the time step for the particle evolution whenever the gradient of the concentration field becomes too large. Practically, it is sufficient to adjust the time step so that the expected length of the particle jump does not exceed the mesh size $\Delta x$.

as $\mathcal{O}(1 / \Delta x)$. Thus for the corresponding particles, the time step may become as small as $\Delta \tau^{(n)} \sim \Delta x^{2}$.

The particle density field $P_{i j}(t)$ is reconstructed from the particle locations as explained in the description to figure 3: each particle contributes fractions of its weight to the four nearest grid points according to the bilinear interpolation rules. This approximation preserves the first moment of the corresponding distribution. It is important to use this kind of moment-preserving approximations, e.g., a simple bin-counting (assigning all weight of a given particle to the nearest grid point) is unsatisfactory. The reason is that this creates an artificial flux towards the grid points: the particles feel their own potentials which become artificially aligned to the nearest grid points. This flux is sufficient to pin singularities to the grid points and disrupt such phenomena as logarithmically weak interaction of singularities with each other and with the boundary of the domain.

The concentration field is propagated according to the implicit second order finite-difference scheme,

$$
\frac{\alpha}{\Delta t}\left[C_{i j}(t+\Delta t)-C_{i j}(t)\right]=\frac{1}{\Delta x^{2}} D_{i j}^{(2)} C(t+\Delta t)-k^{2} C_{i j}(t+\Delta t)+P_{i j}(t),
$$

where $D^{(2)}$ is the standard second difference operator. It is essential to use an implicit scheme for stability reasons because the concentration field acquires logarithmic singularities. This scheme is quite efficient: even though it requires solving a system of linear equations, the matrix of the finite difference operator is symmetric and banded, and may be Cholesky-factorized before the actual computations. The extra computational cost of solving this linear system is then alleviated by relaxation of the $\Delta t \sim \Delta x^{2}$ stability constraint of explicit schemes.

The presented numerical method may be generalized to other similar systems of the form

$$
\begin{aligned}
& \partial_{t} \rho(\boldsymbol{x}, t)=\nabla \cdot(\mu(\boldsymbol{x}, \rho, c) \nabla \rho-\rho \boldsymbol{v}(\boldsymbol{x}, \rho, c)), \\
& \alpha \partial_{t} c(\boldsymbol{x}, t)=\nabla \cdot(G(\boldsymbol{x}) \nabla c)+F(\boldsymbol{x}, \rho, c),
\end{aligned}
$$




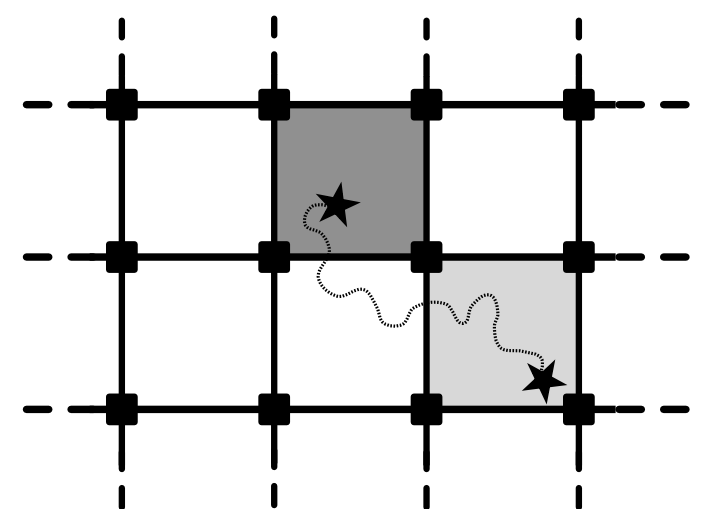

Figure 3. Schematic representation of the numerical scheme. The concentration field $c(\boldsymbol{x})$ is sampled on a uniform grid $(\boldsymbol{\square})$, while the particles $(\star)$ move without any restraints. The particle density $\rho(\boldsymbol{x})$ is reconstructed via bilinear interpolation: each particle contributes fractions of its mass to the four nearest grid points proportionally to the relative distances from the latter, e.g., a particle located at $(x, y)$ within a unit-square grid cell contributes weights proportional to $x y$, $x(1-y), y(1-x)$, and $(1-x)(1-y)$ to the respective grid points.

and to more complicated domains. This makes it a useful tool for studying various aggregation phenomena for which the conventional finite-difference or finite-elements schemes are inefficient.

From the numerical analysis point of view, we do not currently have rigorous estimates of the convergence rates for this scheme. Partially, this problem is complicated due to the singular nature of solutions to the $\mathrm{K}-\mathrm{S}$ equations. In particular, once the blow-ups are formed, one must interpret the K-S PDEs in some proper sense, and while our numerical scheme is able to propagate solutions for all times (i.e. it regularizes the PDEs in some fashion), relation of such numerical solutions to a particular analytical regularization must be carefully investigated. An additional challenge is that one must use some convergence criteria in measure-spaces, e.g., using the Wasserstein metrics, because the regular function-space norms blow up when the particle density acquires atomic components. On the other hand, we could speculate that our method exhibits typical for this kind of schemes errors of orders $\mathcal{O}\left(\Delta x^{2}\right), \mathcal{O}(\sqrt{\Delta t})$, and $\mathcal{O}(1 / \sqrt{N})$ for regular solutions, see e.g., analysis for similar systems in [10]. To estimate the error contributions due to space-time discretization, one could go along the lines of traditional numerical analysis for parabolic PDEs and SDEs, however the dependence on $N$ is more challenging. Thus we perform series of experiments to verify convergence as the number of particles, $N$, becomes large. Figure 4 illustrates these experiments. We vary the number of particles per each grid point of the finite difference discretization, $N_{\mathrm{g}}$, and observe the mismatch that a particular solution has relative to the 'benchmark solution' calculated with $N_{\mathrm{g}}=1024$. We can see that our guess of the $\mathcal{O}(1 / \sqrt{N})$ convergence holds up. We perform a few more numerical experiments illustrating convergence of singular solutions in the end of section 3 , see figure 7 .

\section{Formation and interaction of singularities}

We now concentrate on the elliptic $(\alpha=0)$ case, though some of the reasoning is equally applicable to the parabolic case as well. Generally, one can distinguish the soft and hard blow-ups: in the first case the fields $\rho(\boldsymbol{x})$ and $c(\boldsymbol{x})$ become unbounded but $\rho(\boldsymbol{x})$ does not 


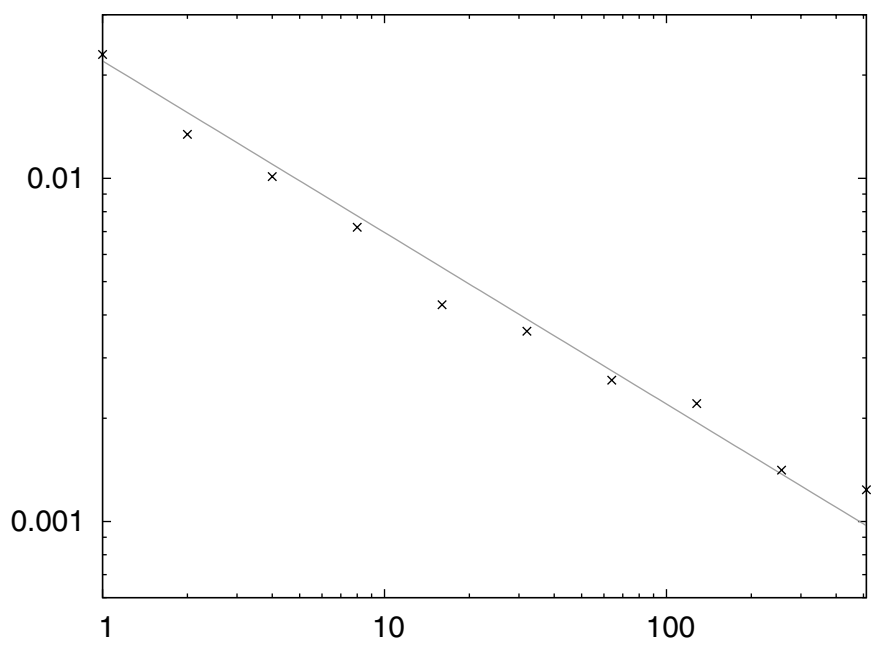

Figure 4. Convergence of the method. The log-log plot displays $L_{1}$ error of the bacteria density, $\rho(\boldsymbol{x})$, as a function of $N_{\mathrm{g}}$-the number of particles per grid point of the finite difference mesh for $c(x)$. The error is computed at time $t=1$, relative to a simulation with $N_{\mathrm{g}}=1024$. The initial datum is a randomly generated smooth density with subcritical mass, $M=0.1$. The computational domain is a unit square, discretized with $\Delta x=0.05$; the other parameters are: $\Delta t=0.001, \alpha=0, k=0.01, \chi=0.1, \mu=0.005$. The crosses depict results of the simulations; the grey line corresponds to the expected error of the order of $1 / \sqrt{N_{\mathrm{g}}}$.

acquire atomic components, while in the second case the particle density field becomes a bona fide distribution. Here we concentrate on the hard blow-ups which have a more apparent physical meaning: atomic components of the particle density correspond to accumulation of particles at some point locations on the given physical length-scale.

It is convenient to study the elliptic model in a slightly different form, as a McK-V system. First, we solve equation $(1 b)$ for $c(x)$ :

$$
c(\boldsymbol{x})=-\int_{\Omega} V_{d}(\boldsymbol{x}, \boldsymbol{y}) \rho(\boldsymbol{y}) \mathrm{d} \boldsymbol{y}=-V_{d} * \rho(\boldsymbol{x}) .
$$

Here $V_{d}(\boldsymbol{x}, \boldsymbol{y})$ is the Green's function for $\Delta-k^{2}$ in $\Omega$, which is assumed to be $d$-dimensional. The entire-space Green's functions (the fundamental solutions) whose singular part is identical to those in bounded domains are known explicitly:

$$
\begin{array}{ll}
V_{1}(\boldsymbol{x}, \boldsymbol{y})=-\frac{\mathrm{e}^{-k|\boldsymbol{x}-\boldsymbol{y}|}}{2 k} & (d=1) ; \\
V_{2}(\boldsymbol{x}, \boldsymbol{y})=-\frac{\mathrm{K}_{0}(k|\boldsymbol{x}-\boldsymbol{y}|)}{2 \pi} & (d=2) ; \\
V_{3}(\boldsymbol{x}, \boldsymbol{y})=-\frac{\mathrm{e}^{-k|\boldsymbol{x}-\boldsymbol{y}|}}{4 \pi|\boldsymbol{x}-\boldsymbol{y}|} & (d=3) .
\end{array}
$$

Here $\mathrm{K}_{0}(\cdot)$ is the modified Bessel function of second kind. Substituting expression (9) for the concentration field $c(\boldsymbol{x})$ into equation ( $1 a)$ we get a closed integro-differential equation for the particle density field,

$$
\partial_{t} \rho(\boldsymbol{x}, t)=\nabla \cdot\left(\mu \nabla \rho+\chi \rho \nabla V_{d} * \rho\right)=\nabla \cdot\left[\rho \nabla \frac{\delta \mathcal{E}}{\delta \rho}\right] .
$$


Here the energy functional is given by

$$
\mathcal{E}(\rho)=\mu \int_{\Omega} \rho(\boldsymbol{x}) \ln \rho(\boldsymbol{x}) \mathrm{d} \boldsymbol{x}+\frac{\chi}{2} \iint_{\Omega} \rho(\boldsymbol{x}) V_{d}(\boldsymbol{x}, \boldsymbol{y}) \rho(\boldsymbol{y}) \mathrm{d} \boldsymbol{x} \mathrm{d} \boldsymbol{y} .
$$

One of the features of the dynamics $(11)$ is that the functional $\mathcal{E}(\rho)$ is non-increasing:

$$
\frac{\mathrm{d} \mathcal{E}}{\mathrm{d} t}=-\int_{\Omega}\left|\nabla \frac{\delta \mathcal{E}}{\delta \rho}\right|^{2} \rho(\boldsymbol{x}, t) \mathrm{d} \boldsymbol{x} \leqslant 0 .
$$

When $\rho(x)$ becomes singular with respect to Lebesgue measure, the first (entropic) term in (12) tends to infinity, which must be compensated by the second (interaction) term. If $d=1$, the Green's function $V_{1}(x)$ is bounded below and so is the interaction term, thus the blow-ups are not possible. This is no longer the case if $d \geqslant 2$ and so the blow-ups are permissible. A more delicate analysis is needed to understand how exactly they are formed.

From standpoint of the stochastic process underlying the Fokker-Planck PDE $(1 a)$, the atomic components of $\rho(\boldsymbol{x})$ correspond to particles aggregating at point locations. Thus the traps which do not allow the particles to escape must be created. Let us first investigate when a particle aggregate creates a trap for a single particle diffusing in its field. Suppose $\rho(\boldsymbol{x})=M \delta(\boldsymbol{x})$, the corresponding concentration field is then proportional to the fundamental solution (10a) and (10b). Equation for the radial component of the location of a single particle diffusing in the field created by $\rho$ is equivalent in law to the following SDE:

$$
\mathrm{d} r_{t}=\left[\chi c^{\prime}\left(r_{t}\right)+(d-1) \frac{\mu}{r_{t}}\right] \mathrm{d} t+\sqrt{2 \mu} \mathrm{d} W_{t} .
$$

For $d=1$ we get

$$
\mathrm{d} r_{t}=-\frac{\chi M}{2} \exp \left(-k r_{t}\right) \mathrm{d} t+\sqrt{2 \mu} \mathrm{d} W_{t} .
$$

This equation is sufficiently regular and does not allow for existence of a trap for any value of $M$, so consistently with energy considerations, no blow-ups are possible in this case. For $d \geqslant 3$, a similar reasoning shows that a trap of an arbitrarily small mass may exist. So the interesting case is $d=2$.

For $d=2$, using that in the leading order, as $r \rightarrow 0, \mathrm{~K}_{0}(r) \sim-\ln (r)$, we obtain the following equation:

$$
\mathrm{d} r_{t}=\left(\mu-\frac{\chi M}{2 \pi}\right) \frac{\mathrm{d} t}{r_{t}}+\sqrt{2 \mu} \mathrm{d} W_{t}
$$

This is a well-known Bessel process [30], it's behaviour near the boundary at $r=0$ depends strongly on the value of $M$. In particular, when

$$
M \geqslant \frac{4 \pi \mu}{\chi}=M_{c}^{*},
$$

the origin is the so-called exit boundary, a trap, i.e., the particles reach it in finite time, and may never escape back to the domain $r>0$. For our problem the implication is that the total mass of at least $M_{c}^{*}$ is needed for existence of a stable singularity in the particle density field. For $M \in\left(0, M_{c}^{*}\right)$, the SDE (16) has the so-called regular boundary at $r=0$. In this case the particles may reach the origin, but are not necessarily trapped there, i.e., may also leave it according to some rules which must be prescribed in addition to the SDE itself. This implies that the original K-S PDEs alone are not sufficient to describe the blow-up dynamics and the exchange of mass between the regular and singular components of the particle density when the mass of the singularity is less than $M_{c}^{*}$.

The critical mass $M_{c}^{*}$ is smallest mass such that a singularity does not shed its mass but only absorbs particles from the smooth component. It is, however, twice less than the mass 
required to create a singularity from smooth initial data. Indeed, consider the $N$-particle SDEs approximating the elliptic $\mathrm{K}-\mathrm{S}$ equations:

$$
\mathrm{d} \boldsymbol{X}_{t}^{(n)}=-\frac{\chi M}{N} \frac{\partial}{\partial \boldsymbol{X}^{(n)}} \sum_{i=1, i \neq n}^{N} V_{d}\left(\boldsymbol{X}_{t}^{(n)}, \boldsymbol{X}_{t}^{(i)}\right) \mathrm{d} t+\sqrt{2 \mu} \mathrm{d} \boldsymbol{W}_{t}^{(n)} .
$$

To study the aggregation dynamics of the particle system, consider the variance of the empirical probability density of the bacteria (4),

$$
R_{t}^{2}=\frac{1}{2 N^{2}} \sum_{i, j=1}^{N}\left|\boldsymbol{X}_{t}^{(i)}-\boldsymbol{X}_{t}^{(j)}\right|^{2}
$$

$R_{t}$ will be referred to as the radius of the system. We concentrate on the problem in the entire space with $k=0$; this corresponds to $V_{2}(\boldsymbol{x}, \boldsymbol{y})=1 /(2 \pi) \ln |\boldsymbol{x}-\boldsymbol{y}|$. Analysis for the other values of $k$, or in the bounded domains is similar (although more technical) because the singularities of $V_{2}(\boldsymbol{x}, \boldsymbol{y})$ are the same in the leading order. In this case, however, there exists an explicit closed equation governing the evolution of $R_{t}$ :

$$
\mathrm{d} R_{t}=\left[2 \mu\left(1-\frac{3}{2 N}\right)-\frac{\chi M}{4 \pi}\left(1-\frac{1}{N}\right)\right] \frac{\mathrm{d} t}{R_{t}}+\sqrt{\frac{2 \mu}{N}} \mathrm{~d} W_{t} .
$$

The driving stochastic process $W_{t}$ obeys the following SDE:

$$
\mathrm{d} W_{t}=\frac{1}{N^{3 / 2} R_{t}} \sum_{i, j=1}^{N}\left(\boldsymbol{X}_{t}^{(i)}-\boldsymbol{X}_{t}^{(j)}\right) \cdot \mathrm{d} \boldsymbol{W}_{t}^{(i)},
$$

and can be shown to be a Wiener process using the Lévy characterization of Brownian motion. We can immediately see that in the limit as $N \rightarrow \infty$, if

$$
M \neq \frac{8 \pi \mu}{\chi}=M_{c}
$$

the evolution of the radius obeys a well-known deterministic equation,

$$
\dot{R}_{t}=-\frac{\gamma}{2 R_{t}}, \quad \gamma=4 \mu\left(M / M_{c}-1\right) .
$$

Hence $R_{t}^{2}=R_{0}^{2}-\gamma t$, and for supercritical masses $\left(M>M_{c}\right)$ the blow-up occurs at time $T=R_{0}^{2} / \gamma$. It is independent of the initial distribution of mass except through $R_{0}$. If $M=M_{c}$, after the time rescaling, equation (20) may be rewritten as

$$
\mathrm{d} R_{t^{\prime}}=\frac{1}{2} \frac{\mathrm{d} t^{\prime}}{R_{t^{\prime}}}+\mathrm{d} W_{t^{\prime}}, \quad t^{\prime}=\frac{2 \mu t}{N} .
$$

This equation describes a critical Bessel process with entrance boundary at $R=0$, in this case $R_{t^{\prime}}$ a.s. never hits zero, although it visits its arbitrarily small neighbourhood. In this limit the particle system remains stochastic even as $N \rightarrow \infty$ and does not approximate the deterministic K-S equations. As $t^{\prime} \rightarrow \infty$, the particle system approaches blow-up infinitely often, but never actually coalesces into a single particle, see [7].

Formation of singularities. Our first numerical experiment is designed to test how well our numerical method predicts the value of the critical mass $M_{c}$ required to develop a singularity from smooth initial data. Notice, first of all, that due to reflection principle for the problem with Neumann boundary conditions, singularity in a corner of the domain, $\Omega=(0, L)^{2}$, only requires a quarter of the critical mass. Since the singularity inside of the domain eventually 

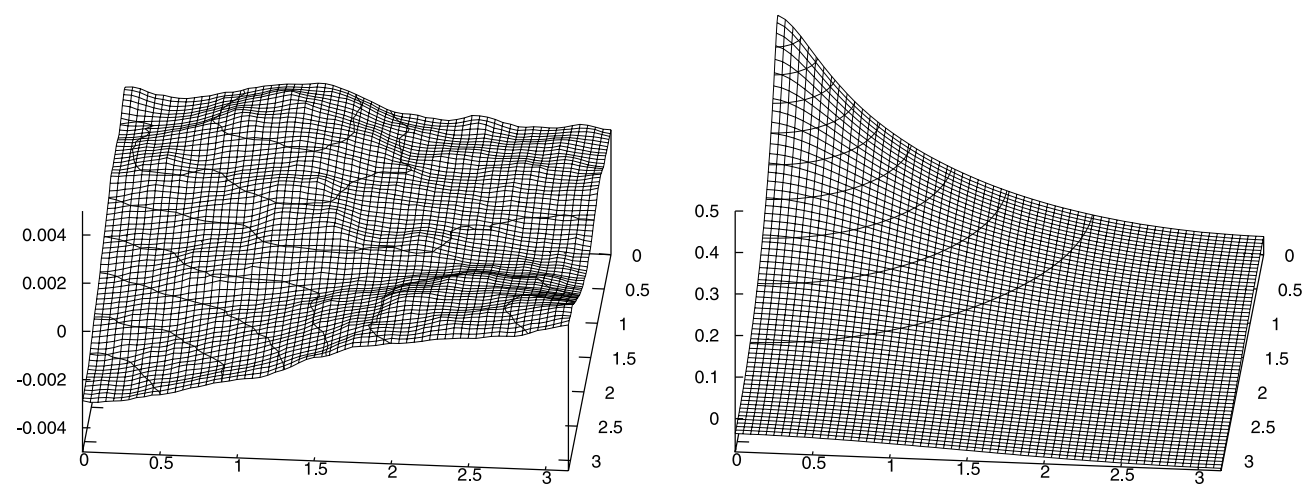

Figure 5. Snapshots $(t=10000)$ of the concentration field $c(x)-M /(k L)^{2}$ demonstrating suband supercritical behaviours in the elliptic $(\alpha=0)$ model. (The constant is subtracted because $c(\boldsymbol{x})$ itself becomes unbounded in a finite domain as $k \rightarrow 0$.) The other parameters are as follows: $k=0.01, \chi=0.1, \mu=0.005 ; \Delta t=0.01, \Delta x=0.05,4 \cdot 10^{3}$ particles. Initially, all particles are distributed randomly with a slight bias towards $(0,0)$. In the supercritical case $(M=0.35$, right plot), a trap is created, while in the subcritical case ( $M=0.34$, left plot), all particles eventually spread uniformly over the entire domain. The theoretical critical mass for the elliptic model with $k=0$ in the entire space is $M_{c}=2 \pi / 5 \approx 1.26$. Notice that only a quarter of the critical mass is needed for a trap in the corner.

migrates into a corner, we perform our experiment in the corner to begin with. We scatter all particles over $\Omega$ with a slight bias towards $(0,0)$ and vary their total mass observing whether the particles remain aggregated, or spread uniformly over the entire domain. The results are presented in figure 5. For the specified values of the parameters, the calculated numerical value of the critical mass lies between 0.34 and 0.35 , while the theoretical prediction for elliptic model with $k=0$ in the entire plane is $M_{c} / 4=\pi / 10$-slightly smaller. The mismatch is a consequence of the simulation in a finite domain, the limit $L \rightarrow \infty, k \rightarrow 0$ is nontrivial because equation $(1 b)$ with Neumann boundary conditions is not well-posed in $\Omega$ when $\alpha=k=0$. In particular, the boundary of the domain far from the corner where the singularity is formed pulls the particles away from the singularity, effectively increasing the critical mass.

Interesting phenomena occur when a singularity with mass $M \in\left(0, M_{c}^{*}\right)$ already exists in the system. In this case the trap absorbs the particles, though the latter may still escape back into the regular component of the particle density. The diffusion process underlying the $\mathrm{K}-\mathrm{S}$ equations is not uniquely defined by its generator and additional rules for behaviour of the particles at singular points must be specified. These rules are not inherently encoded in the $\mathrm{K}-\mathrm{S}$ equations and are related to non-uniqueness of $\mathrm{K}-\mathrm{S}$ regularizations. From the modelling perspective, the exchange of mass between the regular and singular components of the particle density in this regime strongly depends on particular details of the numerical method. Note, however, that if the initial particle density is regular, the smallest possible mass for singularity is greater than $M_{c}=2 M_{c}^{*}$, so this scenario does not occur.

Interaction of singularities is illustrated in figure 6. The singularities attract each other and are also attracted by the boundary of the domain. Equations governing their dynamics may be derived following $[19,43,44]$. If the density $\rho(\boldsymbol{x})$ is purely atomic, we obtain

$$
\dot{\boldsymbol{x}}_{i}(t)=-\chi \frac{\partial}{\partial \boldsymbol{x}_{i}} \sum_{j \neq i} m_{j} V_{2}\left(\boldsymbol{x}_{i}, \boldsymbol{x}_{j}\right) .
$$

Here $m_{j}$-s are the masses of singularities located at $\boldsymbol{x}_{j}$-s, $V_{2}(\boldsymbol{x}, \boldsymbol{y})$ is the Green's function. Note that unlike in the method of Haškovec and Schmeiser [24], in our method, the dynamics of 

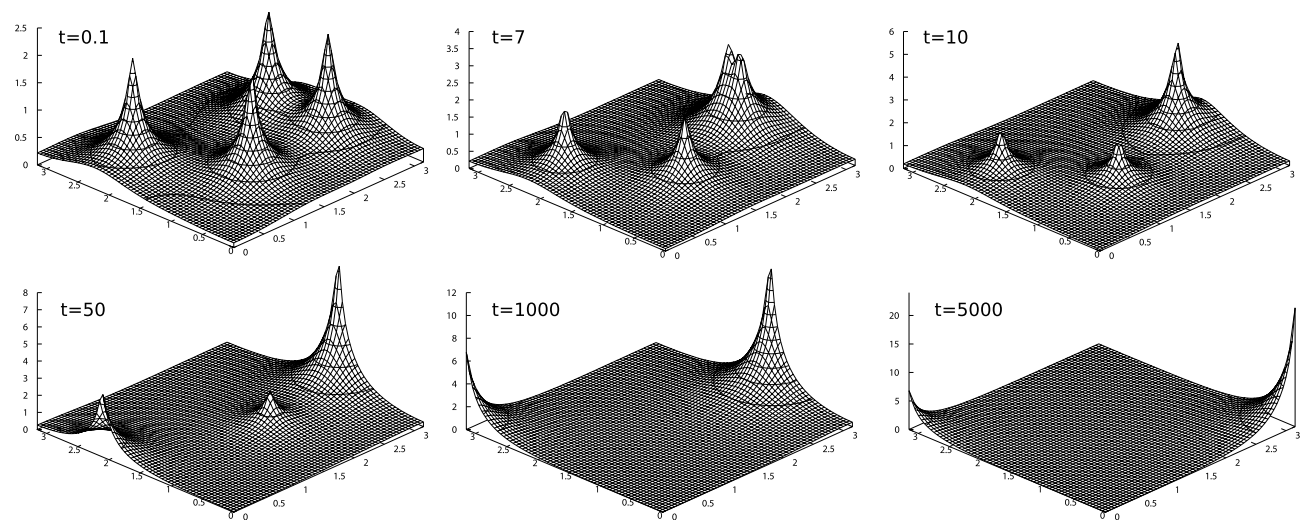

Figure 6. Snapshots of the concentration field $c(x)$ illustrating the motion and interaction of singularities in the elliptic $(\alpha=0)$ model. The other parameters are as follows: $k=1, \chi=0.1$, $\mu=0.005, M=4 ; \Delta t=0.01,4 \cdot 10^{3}$ particles. Initially, the particles are placed around four distinct locations; as time goes on, the singularities are formed, then they merge and travel towards the boundary of the domain, and finally stabilize in the corners. (Notice the scale change for the $z$-axis.)

singularities is not imposed by the numerical method explicitly, i.e., it is a natural consequence of the stochastic particle dynamics.

Equations (25) are identical to equations (3) of the K-S model with particle diffusivity $\mu$ set to zero. The naive reasoning is that once the traps are formed, the particles' diffusion is dominated by the (infinitely strong) drift and ceases to contribute into dynamics: the stochastic dynamics self-averages and the martingale component does not contribute into the mean drift. In order to verify how well our method approximates equations (25), we perform a numerical experiment illustrated in figure 7 . In this experiment we create two singularities far from the boundaries in a sufficiently large domain, so that the interaction potential is well-approximated by the fundamental solution $(10 b)$. (In a bounded domain, the particle interaction potential, $V_{2}\left(\boldsymbol{x}_{i}-\boldsymbol{x}_{j}\right)$, should be replaced by the Green's function which also encodes interaction with the boundary. In particular, this explains attraction of singularities to the boundary and the corners of the domain.) We run the $\mathrm{K}-\mathrm{S}$ solver and track locations of these singularities. Finally, we compare them with locations of the particles evolving according to equation (25) from the same initial data. A near-perfect match indicates that the scheme is very successful in dealing with this kind of phenomena. Observe how the match improves as the number of particles, $N$, increases. In fact, as can be guessed from the formulas similar to equation (20), the numerical solution always retains stochastic effects on the order of $\mathcal{O}(1 / \sqrt{N})$. Only in the limit as $N \rightarrow \infty$ does it become truly deterministic.

\section{Discussion}

Our investigation of the $\mathrm{K}-\mathrm{S}$ equations was motivated by the underlying stochastic particle dynamics. A numerical method based on similar ideas has been recently presented by Haškovec and Schmeiser [24]. The greatest advantage of the H-S method is that the singularities are represented as deterministic particles rather than as a tight cluster of 'elementary' stochastic particles. This, however, requires an explicit knowledge of evolution equations governing the interaction of singularities (at the very least the knowledge of Green's function in a given domain) and is only applicable to the elliptic K-S model. Moreover, the H-S method requires a 


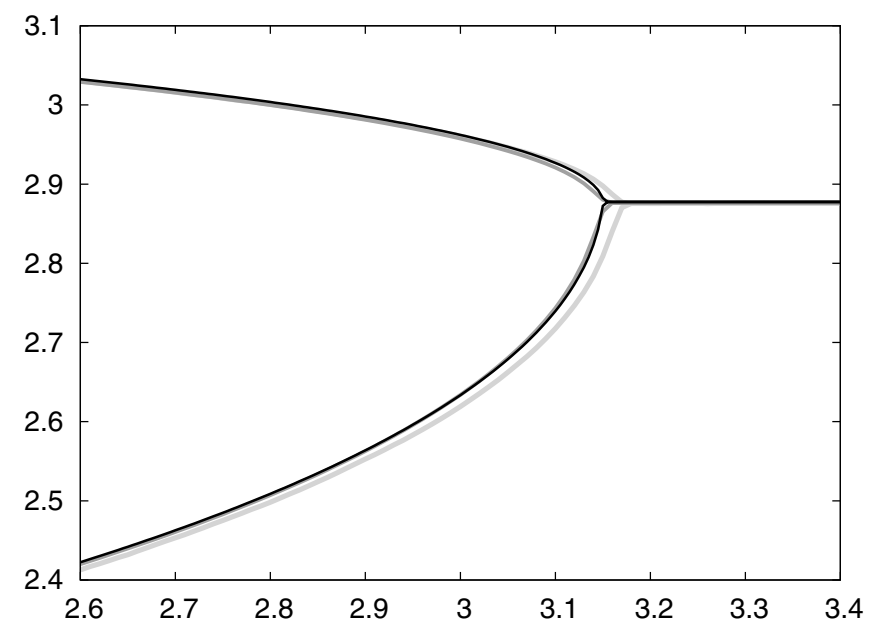

Figure 7. Interaction of singularities in the elliptic $(\alpha=0)$ model. The particle density $\rho(x)$ is initialized with two delta-functions with masses 6.25 and 18.75. These singularities attract each other and eventually merge; their locations are plotted as functions of time $t$. The thin black line corresponds to the simulation of equation (25); the dark grey line - to simulation of the elliptic $\mathrm{K}-\mathrm{S}$ model; $\Delta t=0.01,4 \cdot 10^{5}$ particles are used; the light grey line corresponds to a simulation with $2 \cdot 10^{4}$ particles. The computational domain is chosen sufficiently large so that the boundary effects are negligible on the presented time scale.

direct simulation of an ensemble of interacting particles and thus its computational cost scales as $N^{2}$ with respect to the total number of particles. By combining the particle dynamics and the PDE dynamics of the concentration field which mediates the particle interaction (the PIC ideas) we are able to significantly reduce this computational cost and deal with a much larger number of interacting particles. We can also treat both parabolic and elliptic models with equal ease. The ultimate numerical method should combine ideas presented in this work, the deterministic particles of the $\mathrm{H}-\mathrm{S}$ method, and also high order conventional schemes in the regions where the solution remains regular. Such a multi-model implementation remains a subject for the future work.

Even though the non-uniqueness issue is well-known, the details of how the exit-entrance conditions for the underlying diffusion process are related to the exchange of mass between the singularity and the regular part are not yet well understood. From the modelling perspective, the implication is that a particular numerical scheme provides a regularization of some sort which affects the critical mass $M_{c}^{*}$

Another interesting class of stochastic particle models related to our studies of the $\mathrm{K}-$ $\mathrm{S}$ equations arises in the context of the so-called self-gravitating Brownian particles, see e.g., $[13,14,36]$. We suggest a model which fits into this class and bears an intrinsic relation to the $\mathrm{K}-\mathrm{S}$ model. Consider an ensemble of particles in $d$ dimensions characterized by their masses $m_{n}$ and evolving according to the stochastic differential equations

$$
\mathrm{d} \boldsymbol{X}_{t}^{(n)}=-\chi \frac{\partial}{\partial \boldsymbol{X}^{(n)}} \sum_{k \neq n} m_{k} V_{d}\left(\boldsymbol{X}_{t}^{(n)} \boldsymbol{X}_{t}^{(k)}\right) \mathrm{d} t+\sqrt{2 \mu M / N_{0} m_{n}} \mathrm{~d} \boldsymbol{W}_{t}^{(n)} .
$$

Here $N_{0}$ is the initial number of particles and $M=\sum m_{n}$ is their total mass. If initially $m_{n}=M / N_{0}$, equation (26) is precisely (18). The interaction allows for particle collisions, thus equation (26) is only valid until the first collision, at which point the colliding particles coalesce into a single particle which acquires their combined mass. Dynamics is then restarted 
with the remaining particles. In the limit as $N_{0} \rightarrow \infty$, whenever a particle accumulates an $\mathcal{O}(1)$ mass, its diffusivity becomes zero, i.e., it obeys the deterministic equation (25) which governs evolution of a point singularity in the $\mathrm{K}-\mathrm{S}$ model. Therefore we conjecture that in a proper hydrodynamic limit this system is equivalent to the elliptic $\mathrm{K}-\mathrm{S}$ model. Investigation of this system will be conducted elsewhere.

\section{Acknowledgment}

The author is supported by NSF grants DMS-0807332 and DMS-1056471.

\section{References}

[1] Allen E J and Victory H D Jr 1994 A computational investigation of the random particle method for numerical solution of the kinetic Vlasov-Poisson-Fokker-Planck equations Physica A 209 318-46

[2] Bertozzi A, Carrillo J and Laurent T 2009 Blowup in multidimensional aggregation equations with mildly singular interaction kernels Nonlinearity $22683-710$

[3] Bertozzi A, Laurent T and Rosado J $2011 L^{p}$ theory for the multidimensional aggregation equation Commun. Pure Appl. Math. 64 45-83

[4] Biler P, Corrias L and Dolbeault J 2009 Large mass self-similar solutions of the parabolic-parabolic Keller-Segel model of chemotaxis, arXiv:0908.4493v1 [math.AP]

[5] Birdsall C K 1991 Particle-in-cell charged particle simulations, plus Monte-Carlo collisions with neutral atoms PIC-MCC IEEE Trans. Plasma Sci. 19 65-85

[6] Blanchet A, Calvez V and Carrillo J 2008 Convergence of the mass-transport steepest descent scheme for the subcritical Patlak-Keller-Segel model SIAM J. Numerical Anal. 46 691-721

[7] Blanchet A, Carrillo J and Masmoudi N 2008 Infinite time aggregation for the critical Patlak-Keller-Segel model in $\mathbb{R}^{2}$ Commun. Pure Appl. Math. 61 1449-81

[8] Blanchet A, Dolbeault J and Perthame B 2006 Two-dimensional Keller-Segel model: optimal critical mass and qualitative properties of the solutions Electron. J. Diff. Eqns. 4432

[9] Bossy M and Talay D 1996 Convergence rate for the approximation of the limit law of weakly interacting particles: application to the Burgers equation Ann. Appl. Probab. 6 818-61

[10] Bossy M and Talay D 1997 A stochastic particle method for the McKean-Vlasov and the Burgers equation Math. Comput. 66 157-92

[11] Calvez V and Carrillo J A 2006 Volume effects in the Keller-Segel model: energy estimates preventing blow-up J. Math. Pures Appl. 86 155-75

[12] Carrillo J A, DiFrancesco M, Figalli A, Laurent T and Slepcev D 2010 Confinement in nonlocal interaction equations Nonlin. Anal. Theory Methods Appl. 75 550-8

[13] Chavanis P-H 2007 Critical mass of bacterial populations and critical temperature of self-gravitating Brownian particles in two dimensions Physica A 384392

[14] Chavanis P-H 2007 Exact diffusion coefficient of self-gravitating Brownian particles in two dimensions Eur. Phys. J. B 57391

[15] Chayes L and Panferov V 2009 The McKean-Vlasov equation in finite volume, arXiv:0910.4615v1 [math-ph]

[16] Cottet G H and Raviart P A 1986 On particle-in-cell methods for the Vlasov-Poisson equations Transport Theory Stat. Phys. 15 1-31

[17] Dejak S I, Lushnikov P M, Ovchinnikov Y N and Sigal I M 2012 On spectra of linearized operators for KellerSegel models of chemotaxis Physica D 241 1245-54

[18] Dolbeault J and Perthame B 2004 Optimal critical mass in the two-dimensional Keller-Segel model in $R^{2} C . R$. Math. 339 611-6

[19] Dolbeault J and Schmeiser C 2009 The two-dimensional Keller-Segel model after blow-up Discrete Contin. Dyn. Syst. B 25 109-21

[20] Epshteyn Y 2009 Discontinuous Galerkin methods for the chemotaxis and haptotaxis models J. Comput. Appl. Math. 224 168-81

[21] Epshteyn Y and Izmirlioglu A 2009 Fully discrete analysis of a discontinuous finite element method for the Keller-Segel chemotaxis model J. Sci. Comput. 40 211-56

[22] Epshteyn Y and Kurganov A 2008 New interior penalty discontinuous Galerkin methods for the Keller-Segel chemotaxis model SIAM J. Numer. Anal. 47 386-408

[23] Filbet F 2006 A finite volume scheme for the Patlak-Keller-Segel chemotaxis model Numer. Math. 104 457-88 
[24] Haškovec J and Schmeiser C 2010 Stochastic particle approximation for measure valued solutions of the 2D Keller-Segel system J. Stat. Phys. 135 133-51

[25] Haškovec $\mathbf{J}$ and Schmeiser C 2011 Convergence of a stochastic particle approximation for measure solutions of the 2D Keller-Segel system Commun. Partial Diff. Eqns. 36 940-60

[26] Havlac K 1996 Deterministic and random particle methods applied to Vlasov-Poisson-Fokker-Planck kinetic equations PhD Thesis Texas Tech University

[27] Hortsmann D 2003 From 1970 until now: the Keller-Segel model in chemotaxis and its consequences: I. Jahresber. Deutsch. Math.-Verein. 105 103-65

[28] Hortsmann D 2004 From 1970 until now: the Keller-Segel model in chemotaxis and its consequences: II. Jahresber. Deutsch. Math.-Verein. 106 51-69

[29] Ivanov M F and Shvets V F 1980 The method of stochastic differential equations for computing the kinetics of a plasma with collisions USSR. Comput. Math. Math. Phys. 3 146-55

[30] Karlin S and Taylor H M 1981 A Second Course in Stochastic Processes (New York: Academic)

[31] Keller E F and Segel L A 1970 Initiation of slime mold aggregation viewed as an instability J. Theor. Biol. 26 399-415

[32] Lushnikov P M 2010 Critical chemotactic collapse Phys. Lett. A 374 1678-85

[33] McKean H P 1967 Propagation of chaos for a class of nonlinear parabolic equations (Lecture Series in Differential Equations vol 7) (Washington DC: Catholic University) pp 41-57

[34] Patlak C S 1953 Random walk with persistence and external bias Bull. Math. Biol. 15 311-38

[35] Saito N 2007 Conservative upwind finite-element method for a simplified Keller-Segel system modelling chemotaxis IMA J. Numer. Anal. 27 332-65

[36] Sire C and Chavanis P-H 2010 Collapse and evaporation of a canonical self-gravitating gas, arXiv:1003.1118

[37] Stevens A 2000 The derivation of chemotaxis equations as limit dynamics of moderately interacting stochastic many-particle systems SIAM J. Appl. Math. 61 183-212

[38] Strehl R, Sokolov A, Kuzmin D, Hortsmann D and Turek S 2011 A positivity-preserving finite element method for chemotaxis problems in 3D, preprint 417, TU-Dortmund

[39] Strehl R, Sokolov A, Kuzmin D and Turek S 2010 A flux-corrected finite element method for chemotaxis problems Comput. Methods Appl. Math. $10219-32$

[40] Sznitman A 1989 Topics on Propagation of Chaos (Lecture Notes on Mathematics vol 1464) ed P L Hennequin (Berlin: Springer) pp 165-250

[41] Tindall M J, Maini P K, Porter S L and Armitage J P 2008 Overview of mathematical approaches used to model bacterial chemotaxis: I. The single cell Bull. Math. Biol. $701525-69$

[42] Tindall M J, Maini P K, Porter S L and Armitage J P 2008 Overview of mathematical approaches used to model bacterial chemotaxis II: Bacterial populations Bull. Math. Biol. 70 1570-607

[43] Velázquez J J L 2004 Point dynamics in a singular limit of the Keller-Segel model: I. Motion of the concentration regions SIAM J. Appl. Math. 64 1198-223

[44] Velázquez J J L 2004 Point dynamics in a singular limit of the Keller-Segel model: II. Formation of the concentration regions SIAM J. Appl. Math. 64 1224-48

[45] Victory Jr H D and Allen E J 1991 The convergence theory of particle-in-cell methods for multidimensional Vlasov-Poisson systems SIAM J. Numer. Anal. 28 1207-41

[46] Villani C 1992 Topics in Optimal Transportation (Graduate Studies in Mathematics vol 58) (Providence, RI: American Mathematical Society)

[47] Wallman S 2000 On the approximation of the Vlasov-Poisson system by particle methods SIAM J. Numer. Anal. 37 1369-98 